\title{
Verstärkung aversiver Alkoholwirkungen
}

Fragestellung: Wie beeinflusst der Single-Nucleotid-Polymorphismus (SNP) von Asn40Asp die pharmakogenetischen Effekte von Naltrexon bei Probanden asiatischer Herkunft?

Hintergrund: Neuere klinische und experimentelle Untersuchungen haben gezeigt, dass die Wirksamkeit von Naltrexon in der Rückfallprophylaxe bei Alkoholabhängigkeit durch den Asn40Asp SNP des $\mu$-opioid-Rezeptor-Gens (OPRM1) beeinflusst wird. Vor diesem Hintergrund weisen heterozygote Asp40-Träger stärkere Effekte durch Einnahme von Alkohol in Bezug auf subjektive Intoxikation, Sedierung und Stimulation auf. Die Allelfrequenzen dieses Polymorphismus variieren jedoch erheblich in Abhängigkeit des ethnischen Hintergrundes, die Asp40-Frequenz ist bei Menschen asiatischer Herkunft deutlich häufiger (50\%) als beispielsweise bei Kaukasiern (20\%).

Patienten und Methodik: Für die doppelblinde, randomisierten und placebokontrollierte Studie wurden 35 amerikanische College-Studenten asiatischer Herkunft rekrutiert. Es handelte sich dabei um regelmäßig stark trinkende Probanden, die sich nicht in medizinischer Behandlung befanden und keine Abhängigkeitsdiagnose aufwiesen. Nach der Einnahme von Naltrexon

Ray LA, Spencer B, Chin PF, Miotto K. Pharmacogenetics of naltrexone in asian americans; $\mathrm{A}$ randomized placebo-xontrolled Laboratory Study. Neuropsychopharmacology 2012; 37: $445-55$ oder Placebo wurde eine intravenöse Ethanolinfusion appliziert. Primäre Zielparameter dieser Untersuchung waren subjektive Intoxikation und Alkohol-Craving, die mithilfe von standardisierten Fragebögen erfasst wurden.

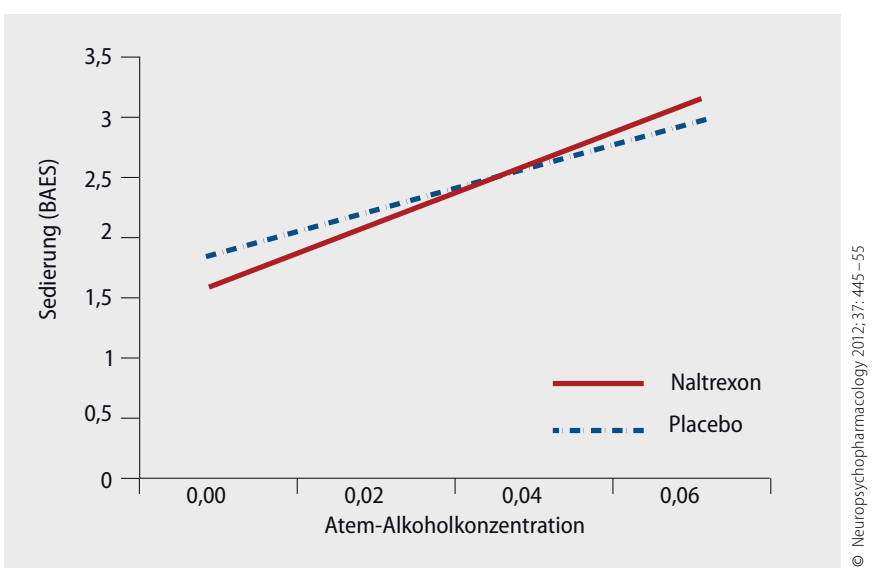

1 Sedierung bei homozygoten Asn40 Allelträgern

Ergebnisse: Im Vergleich zu Placebo und $\mathrm{zu}$ homozygoten Asn40-Allelträgern (Abb. 1) zeigten sich bei den Trägern des Asp40 -Allels, die Naltrexon eingenommen hatten, ausgeprägtere alkoholinduzierte Effekte bei Sedierung, subjektiver Intoxikation und Alkohol-Craving. Interessanterweise waren Genotypeffekte auf Stimulation nicht nachzuweisen.

Schlussfolgerungen: Auch in der Gruppe von Asp40-Allelträgern asiatischer Herkunft konnte eine verbesserte Naltrexonresponse gezeigt werden. Diese Wirksamkeit bezog sich insbesondere auf die Verstärkung aversiver Wirkungen des Alkohols und Craving, nicht jedoch auf Stimulationseffekte.

\section{-Kommentar von Philippe Pfeifer und PD Dr. Christoph Fehr}

\section{Wichtige Einblicke in biobehaviorale Mechanismen}

Die vorliegende Arbeit beantwortet wichtige Fragen zu biobehavioralen Mechanismen der Alkohol- und Naltrexonwirksamkeit. Als Hauptergebnis zeigte sich im Verumarm der vorliegenden Population neben der Abnahme des AlkoholCravings eine verstärkte Wahrnehmung subjektiver Intoxikation unter Naltrexon, was dem Erleben einer aversiven Reaktion entspricht. Eine herabgesetzte Stimulation durch Alkohol bei Naltrexoneinnahme, wie in Untersuchungen an kaukasischen Probanden und Patienten gezeigt, konnte hier nicht nachgewiesen werden. Dies stützt die Hypothese, nach der Naltrexon bei Menschen asiatischer Herkunft eher durch die Verstärkung aversiver Alkoholwirkung als durch die Dämpfung stimulierender und belohnender Alkoholeffekte wirkt [1].

Die Studie weist mit einem randomisierten, doppelblinden und placebokontrollierten Studiendesign einen JADAD-Score von 4 auf und erreicht damit einen hohen Qualitätsstandard. Als Schwachpunkt sollte die Heterogenität der Population genannt werden, da sowohl Frauen als auch Männer eingeschlos- sen wurden. Des Weiteren ist zu bemängeln, dass bezüglich der Alkoholinfusionen keine Placeboinfusion durchgeführt wurde und somit hier keine doppelte Verblindung erfolgte.
Referenz
1. Eng MY. Alcohol Res Health 2007; 30: 22-7

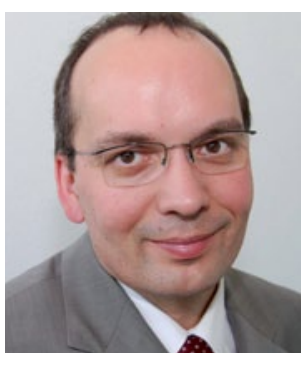

PD Dr. med. Christoph Fehr, Frankfurt am Main

Chefarzt der Klinik für Psychiatrie, Psychotherapie und Psychosomatik, Markus-Krankenhaus, Frankfurt am Main E-Mail: christoph.fehr@fdk.info 